\title{
Application of a Coupled Simulation-Optimization System Called AnyPLOS in a Cold Foam Production Line
}

\author{
Mohammad Amin Jahanpour, Kamran Farnian, and Kourosh Tahouri
}

\begin{abstract}
A prototype simulation-optimization system called AnyPLOS, which couples an Artificial Neural Network (ANN) based simulation model with a genetic algorithm optimization model, is presented. AnyPLOS is designed to discover value of effective input parameters of a production line so that all required quality control tests on the output product is satisfied. First an ANN was trained and tested to provide an acceptable level of accuracy in prediction of production line outputs, and then it was coupled with a GA optimization module to find desired solutions. A real world case study, in Erish Khodro manufacturing company, was set up and the foam production process input parameters were optimized so that the produced samples satisfied quality requirements. In order to verify the results, discovered solutions were used to produce real foam samples in the production line. After that, quality control tests were performed on samples. Quality test results were, as predicted by ANN, within the desired range. In order to estimate the performance of the trained ANN, experimental observations were compared to values which were predicted by ANN. A convincing correlation was found between ANN predictions and experimental values.
\end{abstract}

Index Terms-AnyPLOS, genetic algorithm, neural network, cold foam, production line.

\section{INTRODUCTION}

Estimating various quality aspects of outputs of a production line has always been a challenge in process based industries. Produced samples are supposed to satisfy specific quality control criteria. That is when arbitrary or unexpected alterations in raw materials or in other effective input parameters influence quality aspects of the output product.

The knowledge to predict the resulting outcome as a function of changes in input parameters is an absolutely valuable tool in order to look for a set of input parameters which result in the production of outputs with acceptable or desirable quality aspects.

'Trial and Error' procedure has widely been used as a traditional method to manufacture a product which meets all quality control requirements. However, despite the high costs and time consumption, this method does not often provide reliable solutions. Procedural-based production lines sustain significant costs during 'Trial and Error' procedure. That is when a modern simulation-optimization system may provide reliable solutions and eventually reduce the production costs,

Manuscript received January 24, 2013; revised March 30, 2013.

M. Jahanpour was with Iran University of Science and Technology, Tehran, Iran (e-mail: ajahanpur@civileng.iust.ac.ir).

K. Farnian and K. Tahouri were with Central Branch of Islamic Azad University, Tehran, Iran. (e-mail: farnian@sabalift.com, kourosht50@gmail.com). time consumption or wastes.

This paper describes a prototype simulation-optimization system called AnyPLOS ${ }^{1}$. Coupling an Artificial Neural Network (ANN) based simulation model with a Genetic Algorithm (GA) optimization model is used to find effective input parameters which result in an output product which satisfies all pre-specified quality control tests.

In the following sections, AnyPLOS procedure is described first, then the simulation and optimization modules are thoroughly discussed and finally a real-world case study of AnyPLOS application in a cold foam production line is illustrated. Future works section is also presented to explain ideas for AnyPLOS future developments.

Two acronyms which appear in this paper many times are IP and QA. An IP, standing for Input Parameter, refers to an adjustable parameter which if changed, will influence quality aspects of the output product of a production line. QA, standing for Quality Aspect, is a quality corresponding value which is determined for a product by quality control experiments.

AnyPLOS process initiates by determining IPs and QAs of a production line. First, it should be explicitly determined that which QAs of the output product is about to be controlled by quality control tests. Then a holistic investigation is needed to detect which parameters (IPs) may influence those QAs. Once detected, IP and QA arrays are defined.

Next step, as it will be thoroughly discussed in simulation section, is to use the production line to product some samples and perform quality control tests on the outputs. Once the simulation module is proved to provide an acceptable level of accuracy in prediction of production line outputs, it will be coupled with a GA optimization module. Optimization module triggers a heuristic search procedure to locate optimum IP arrays in the decision space. Solutions found after the search procedure, are IP arrays which if be applied to the production line, QAs of the output product shall satisfy quality control tests. Although all achieved solutions are supposed to result in the production of an acceptable product, however they may have different characteristics. Some may reduce production costs and some may decrease time consumption or wastes. Manager may select the solution which is more close to his/her production policies.

\section{Methodology}

\section{A. Simulation}

A simulation module is required to accurately estimate the

${ }^{1}$ Any Production Line Optimization System (www.anyplos.com) 
QA array of an output product which is manufactured based on a specific IP array. A particular simulation method such as discrete event simulation, or a combination of several modeling approaches may be most appropriate dealing with different production lines. However, AnyPLOS is supposed to be a procedure-independent system, targeting various types of production lines. So the simulation module should capture correlations between variables of a production line that analytically relate. An Artificial Neural Network (ANN) is proved to be able to derive meaning from complex and even fairly inaccurate data. ANN is used to extract patterns and identify trends that are too complicated to be found by either humans or regular computer procedures [1]. A well trained ANN can be considered as an expert a production line, who based on years of experiences, initiatively knows the answer for any what if question.

ANNs have been used to solve numerous problems associated with manufacturing operations. Application of ANN in manufacturing system design [2]-[6], manufacturing process control [7], robot scheduling [8], industrial pattern recognition [9], and manufacturing operational decision [10] is reported.

A back-propagation ANN to estimate the QA array corresponding to any IP array is developed. The ANN consists of an input layer (IP array), an output layer (QA array) and adjustable number of intervening layers. These also called "hidden layers" can capture the non-linear relationship between variables.

The ANN should be trained with experiments so that a sufficient level of accuracy is obtained. Every training experiment is a distinctive sample created in a production line including both IP array and the resulting QA array for that sample. To create an experiment, a set of IPs is defined. Based on that IP arrays, samples are manufactured and then quality control tests are conducted to produce QA arrays for experiments.

DOE (Design Of Experiments), a sampling method which is highly recommended by quality management standards such as QS9000, is employed to produce the minimum number of instances. DOE provides an essential but certainly not enough number of experiments. More experiments are required to probe the decision space of the problem and improve training of the ANN. Having gathered all experiments, the ANN is trained employing the back-propagation training algorithm which is best-known supervised learning approach for ANNs [11].

\section{B. Optimization}

It should be noted that production line models are often known as non-convex, non-linear programming problems. In the case of highly non-linear optimization problems where the convexity of the objective function and feasible region cannot be ensured, classical optimization techniques are not guaranteed to find solutions. Furthermore, being gradient-based, classical optimization techniques consume much CPU time computing derivatives. In recent years, researchers employed heuristic optimization techniques that do not require computing derivatives and are proven to be more efficient in converging to solutions. GA, developed by Holland in 1973 [12], is considered to be a powerful and robust heuristic search algorithm for non-linear optimization of non-linear and non-convex problems. GA uses natural rules of survival in pursuit of the ideas of adaptation to look for optimal solutions of complex problems. GA is able to optimize discrete or continuous variables, escape from local optima, search a broad area of the decision space, and address a large number of variables without requiring objective function derivative computations.

GA commences using an initial population of individuals (called chromosome) including parameters (called gene) which are randomly selected within the range of decision parameters. In this study, a chromosome, as a potential solution of the problem, stands for an IP array and includes genes which correspond IPs. Genes are decision parameters which are to be optimized. In every evolutionary step (called generation), the chromosomes in the current population are evaluated according to the objective function. This is when the ANN based simulation model is called to estimate the QA array for the chromosome and based on that, objective function for every chromosome in the population is determined. To form the next generation, chromosomes are reproduced from the old generations by genetic operators (selection, crossover, and mutation).

During the reproduction phase, individuals with the best fitness values in any generation (elites) are guaranteed to continue to exist in the next generation. Simulated Binary Crossover method developed by Deb and Agrawal [13] is used as crossover operator. The real-coded GA evolution is continued until a stopping criterion is met. Predefined stopping criteria include maximum number of generations, time limit, and stall generations. GA flowchart is demonstrated in Fig. 1.

The objective function (1) is based on minimizing the violation of constraints. Model constraints include IPv and QAv which are sum of IPs and QAs violations from the assigned ranges of variation respectively. In other words, IPs and QAs values are checked to be between a minimum and a maximum value. If any IP or QA has a value outside its range, then the amount of error will be added to IPv (2) or QAv (3) respectively and finally these two values together form the objective function of the chromosome.

$$
\begin{gathered}
\text { Minimize } \quad f=I P v+Q A v \\
I P v=\sum_{i=1}^{I P n} \max \left(0, I P(i)-I P_{\max }(i)\right) \\
+\max \left(0, I P_{\min }(i)-I P(i)\right) \\
Q A v=\sum_{i=1}^{Q A n} \max \left(0, Q A(i)-Q A_{\max }(i)\right) \\
+\max \left(0, Q A_{\min }(i)-Q A(i)\right)
\end{gathered}
$$

In above equations, IPn and QAn are number of IPs and QAs in a production line, respectively. Equation (2) states that any IP may vary within a pre-specified range which is determined by user. IP ranges may be defined considering economic features. For example, if a manager thinks that an IP directly affects the cost of production, he/she can manually limit the IP to small values so AnyPLOS looks for feasible solutions which excel in quality control tests and yet limits 
the specific IP within the desired range. Equation (3) describes limitations applied to QAs of the production line output. These criteria may be determined so that the quality control requirements are satisfied. However they may be adjusted to look for desired solutions which may result in less production costs, less time consumption, or less wastes.

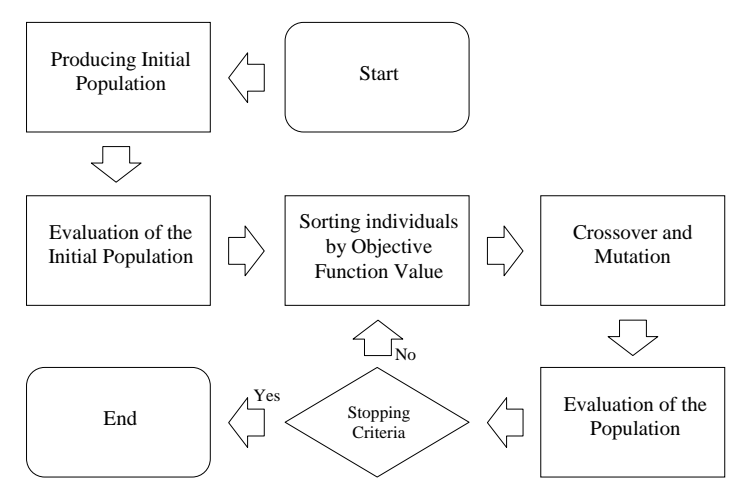

Fig. 1. Flowchart of the optimization module.

Initiating from different random populations, GA is run multiple times to find solutions. GA is most likely to find solutions, only if the user-defined criteria for IPs and QAs are determined so that possible solutions inside the decision space actually exist. After multiple runs, a set of solutions, each provide IPs supposed to result to an acceptable output product, is built.

The proposed Simulation-Optimization model performs an iterative and heuristic procedure in which the GA optimization model calls the simulation model to evaluate the objective function of each solution. The search process continues until one of predetermined stopping criteria is reached.

\section{CASE STUDY}

In this section, a recent application of AnyPLOS in foam production industry is illustrated.

Erish Khodro manufacturing company, located in Qazvin industrial zone in Iran, produces cold cure seating foam which has a wide use in car seats in automotive industry. The foam production line benefits from technology needed for producing dual hardness foams.

QAs of the produced foam are Compression Set, Tensile Strength, Elongation, Tear, and Compression Load Deflection (CLD). These five QA are inspected by quality control tests and the results are compared with corresponding allowed ranges.

Compression set percent represents a measure of the permanent deformation of foam after being compressed between two rigid plates for a specific time and temperature condition. Maximum allowed value of compression set result is $15 \%$. Tensile strength measures the force required to break a specific area of foam as it is pulled apart. Tensile strength experiment is passed if result is not less than $70 \mathrm{Kpa}$. The extent to which the foam can be stretched before it breaks is measured by elongation and is expressed as a percentage of original length of the foam. Results greater than $90 \%$ are acceptable in this test. Tear strength measures of the force required to continue a tear in foam after the appearance of a split. Minimum allowed value of tear strength result is 140 $\mathrm{N} / \mathrm{m}$. CLD is a test method to determine load bearing capacity and is expressed in force per area at a given percent deflection of the foam. In this case study, CLD values between $8.7 \mathrm{Kpa}$ and $10.3 \mathrm{Kpa}$ are acceptable. These five measures form the QA array of this production line as \{Compression set, Tensile, Elongation, Tear, CLD\}

Effective IPs of the production line which are believed to influence the aforementioned QAs of the produced foam were found to be: mixture density (D) in $\mathrm{Kg} / \mathrm{m}^{3}$, ratio-percent of isocyanates to polyols amount in mixture (R), polyols's mixing pressure (Pp) in bar, isocyanates's mixing pressure (Pi) in bar, and curing time of the mixture $(\mathrm{T})$ in minutes. So an IP array is considered as $\{\mathrm{D}, \mathrm{R}, \mathrm{Pp}, \mathrm{Pi}, \mathrm{T}\}$. IPs may change within specific ranges which are determined by experts of foam production. IPs ranges along with quality criteria for QAs are presented in Table I.

TABLE I: ACCEPTABLE RANGES

\begin{tabular}{ccc|ccc}
\hline \hline \multicolumn{3}{c|}{ Acceptable Ranges of IPs } & \multicolumn{3}{c}{ QAs Criteria } \\
\hline Min & IP & Max & Min & QA & Max \\
\hline 42.6 & $\mathrm{D}\left(\mathrm{Kg} / \mathrm{m}^{3}\right)$ & 61.57 & $0 \%$ & Compression set & $15 \%$ \\
48 & $\mathrm{R}(\%)$ & 70 & 70 & Tensile (Kpa) & - \\
100 & $\mathrm{P}_{\mathrm{p}}(\mathrm{bar})$ & 185 & $90 \%$ & Elongation & - \\
100 & $\mathrm{P}_{\mathrm{i}}$ (bar) & 160 & 140 & Tear (N/m) & - \\
3 & $\mathrm{~T}$ (minute) & 4.5 & 8.7 & CLD (Kpa) & 10.3 \\
\hline \hline
\end{tabular}

DOE, as discussed in simulation section, considers all possible combinations of high and low levels for each IP and generates $2^{\mathrm{IPn}}$ samples of experiment (IPn is number of IPs). In this case, 32 samples are produces by DOE $\left(2^{\mathrm{IPn}}=2^{5}\right) .8$ more instances of most commonly applied IPs in the history of the production line were added to the list of experiments to improve the training of the ANN. All of the 40 samples were requested to be produced in the production line. Quality control tests were performed on output foams. QA array of each IP array was gathered based on experimental results.

ANN was trained by IP arrays as input and QA arrays as output. Fig. 1 shows the topology of the ANN which is designed by try and error. Back-propagation training procedure of ANN continued until the coefficient of determination for learning data reached to 0.98 .

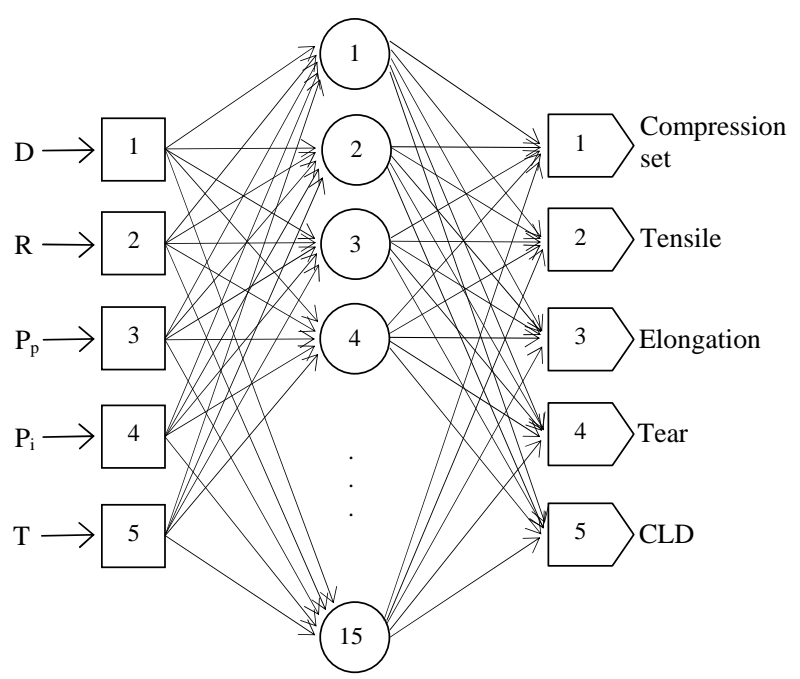

Fig. 2. Topology of the ANN. 
Results show that the ANN estimates every QA of foam samples in an acceptable level of accuracy. So as the simulation module, the trained ANN is ready to be coupled with the GA optimization module. As discussed in optimization section, objective function of GA is defined to be a sum of violations amount in constrains. Zero value of the objective function, which is considered as the optimum condition, only occurs when all constraints are regarded. An optimum solution refers to a condition when all IPs and QAs values are within their predefined range. It means that the optimization module has found an acceptable IP array which the simulation module estimates the corresponding QAs to be in the predefined range. Based on (1) to (3), and ranges defined in table 1 , objective function of the optimization problem is formulated. Multiple runs were performed and several solutions with zero value of objective function were achieved. Fig. 3 presents progressive evolution of the objective function for one of the optimum solutions. With a population size of 20 , nearly 80 generations were required to obtain a zero value objective function.

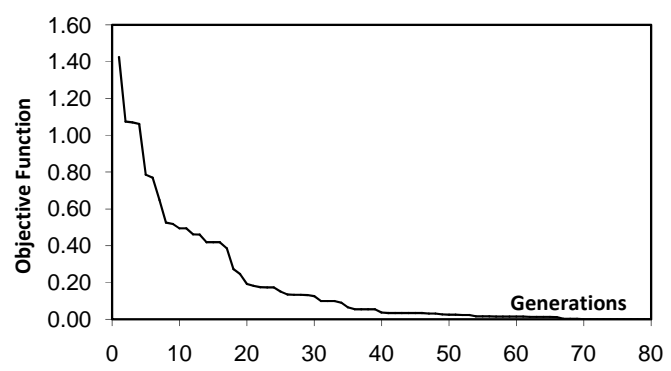

Fig. 3. Progressive evolution of the objective function for a sample solution.

Solutions were investigated and four distinguish solutions, which the other solutions were barely different from them, were detected. These four solutions which are presented in Table II, could be considered as key states while the other solutions are found to be similar to them.

TABLE II: FOUR MAIN OPTIMUM SOLUTIONS

\begin{tabular}{lllll}
\hline \hline \multicolumn{1}{c}{ IP } & Solution \#1 & Solution \#2 & Solution \#3 & Solution \#4 \\
\hline $\mathrm{D}\left(\mathrm{Kg} / \mathrm{m}^{3}\right)$ & 58.010 & 59.337 & 56.700 & 52.529 \\
$\mathrm{R}(\%)$ & 128.103 & 125.047 & 131.283 & 101.422 \\
$\mathrm{P}_{\mathrm{p}}$ (bar) & 182.397 & 163.544 & 142.573 & 182.199 \\
$\mathrm{P}_{\mathrm{i}}$ (bar) & 54.026 & 51.025 & 54.765 & 58.768 \\
$\mathrm{~T}$ (minute) & 4.014 & 3.396 & 3.885 & 3.080 \\
\hline \hline
\end{tabular}

The four IP sets were used to produce four foam samples in the production line. Quality control tests were also performed on the four produced foam samples to determine experimental QA arrays. Experimental results, which are presented in "Obs" columns of Table III, show that all four foam samples are acceptable.

TABLE III: EXPERIMENTAL OBSERVATION VERSUS ANN PRICITION

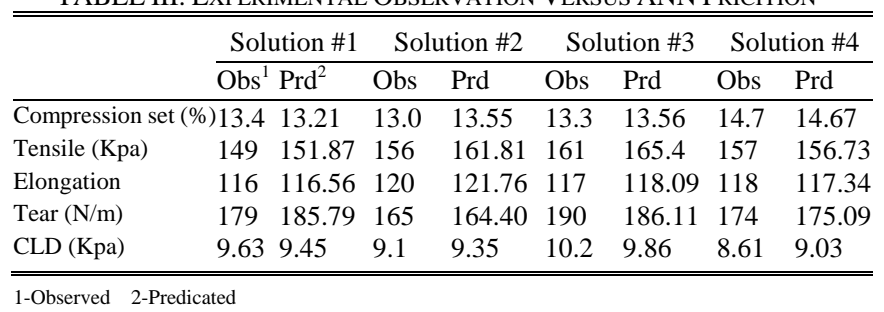

1-Observed 2-Predicated
In order to verify the results, experimental observations were compared to QA arrays which were predicted by ANN. Fig. 4 to Fig. 8 present the correlation between experimental and predicted values of compression set, tensile, elongation, tear, and CLD tests.

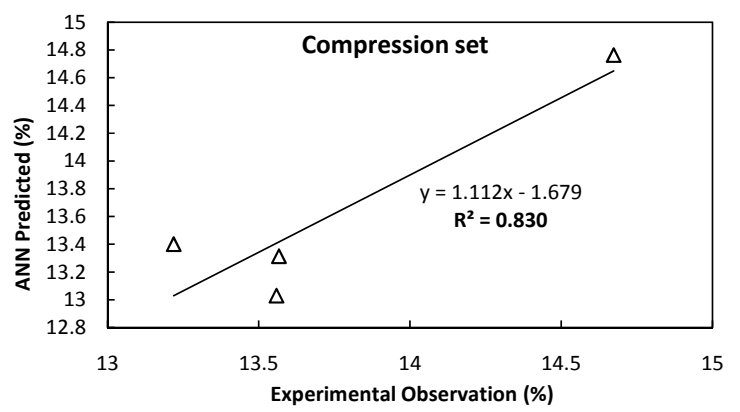

Fig. 4. Correlation between predicted and observed values of compression set test.

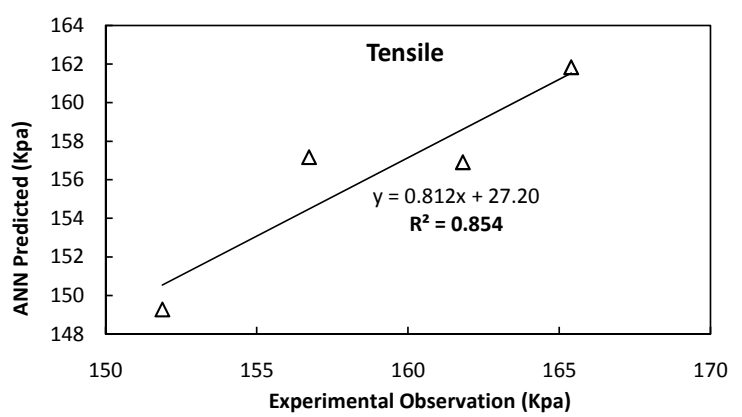

Fig. 5. Correlation between predicted and observed values of tensile test.

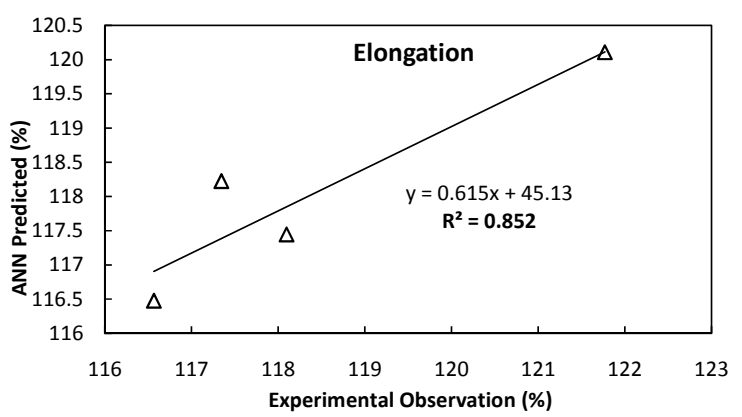

Fig. 6. Correlation between predicted and observed values of elongation test.

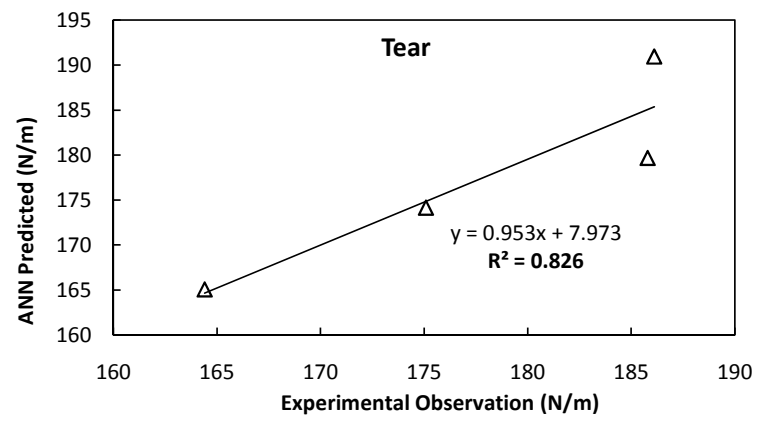

Fig. 7. Correlation between predicted and observed values of tear test.

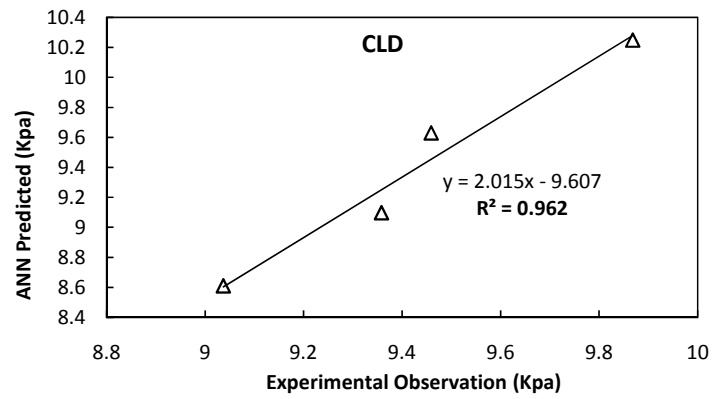

Fig. 8. Correlation between predicted and observed values of CLD test. 
A convincing correlation is found between observed and ANN predicted QAs. All four foam samples which were produced with IPs found by AnyPLOS (Table 2), satisfied quality control tests.

\section{FUTURE WORKS}

The acquired set of solutions from the optimization step may satisfy demands of some managers as they may only look for IPs which result in productions with acceptable QAs. But some of those solutions will certainly reduce production costs, time consumption and wastes. AnyPLOS can help managers to wisely discriminate between the acquired IP arrays in order to find one which best fits expectations. IPs which affect production costs, time consumption, or wastes can be selected as attributes to make such a decision. A multiple attribute decision making algorithm may be added to the prototype of AnyPLOS for this purpose.

Another modification to the prototype of AnyPLOS may be applied in the optimization module. Cost functions may be considered as objective function to be minimized and IPs ranges may be thought as model constraints. Such a configuration requires GA to solely look for a global optimum solution. That unique solution is guaranteed to pass all quality tests while production costs are kept as low as possible. This modification to AnyPLOS is possible only if cost equations are extracted from the production line by a holistic commercial investigation on the production process.

\section{REFERENCES}

[1] R. Wilson and R. Sharda, Neural Networks, OR/MS TODAY, A Joint Publication of ORSA and TIMS, 1992, pp. 36-42.

[2] Y. B. Moon and S. C. Chi, "Generalized part family formation using neural network techniques,” Journal of Manufacturing Systems, vol. 1, no. 3 , pp. 149-159.

[3] S. Kaparthi and N. C. Suresh, "Machine-component cell formation in group technology: a neural network approach,” International Journal of Production Research, vol. 30, no. 6, pp. 1353-1367, 1992.

[4] H. Lee, C. O. Malave, and S. Ramachandran, "A selforganizing neural network approach for the design of cellular manufacturing systems," Journal of Intelligent Manufacturing, vol. 3, pp. 325-332, 1992.

[5] Y. B. Moon, "Establishment of a neurocomputing model for part family/machine group identification," Journal of Intelligent Manufacturing, vol. 3, pp. 173-182, 1992.

[6] B. Wu, "An introduction to neural networks and their applications in manufacturing," Journal of Intelligent Manufacturing, vol. 3, pp. 391-403, 1992.
[7] D. F. Cook and R. E. Shannon, "A predictive neural network modeling system for manufacturing process parameters," International Journal of Production Research, vol. 30, no.7, pp. 1537-1550, 1999.

[8] Y. Yih, T. P. Liang, and H. Moskowitz, "Robot scheduling in a circuit board production line: a hybrid or/ann approach," ZZE Transactions, vol. 25, no. 2, pp. 26-33, March 1993.

[9] Y. Yao, W. J. Freeman, B. Burke, and Q. Yang, "Pattern recognition by a distributed neural network: an industrial application," Neural Networks, vol. 4, pp. 103-121, 1991.

[10] Z. N. Mevawalla, G. S. May, M. Honjo, and M. W. Kiehlbauch, "Neural network modeling of fabrication yield using manufacturing data," in Proc. Advanced Semiconductor Manufacturing Conference (ASMC), 22nd Annual IEEE/SEMI, 2011, pp. 1-6.

[11] F. Zahedi, "An introduction to neural networks and a comparison with artificial intelligence and expert systems," Znterfnces, vol. 21, no. 2, pp. 25-38, April 1991.

[12] J. H. Holland, "Genetic algorithms and the optimal allocation of trials," SIAM Journal on Computing, vol. 2, no. 2, pp. 88-105, June 1973.

[13] K. Deb and R. B. Agrawal, "Simulated Binary Crossover for Continuous Search Space,” Complex Systems, vol. 9, pp. 115-148, 1995.

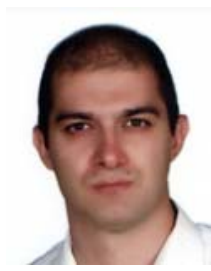

Mohammad Amin Jahanpour received his M.Sc. degree in civil engineering in the area of design of hydraulic structures in 2011 from Iran University of Science and Technology (IUST), Tehran, Iran. His research activities include optimum design and operation of conjunctive water resources systems, simulation and optimization of industrial procedures, evolutionary optimization algorithms, system dynamics, and artificial neural networks. Publications, certificates and developed scientific programs are presented in his personal webpage at aminjahanpour.ir.

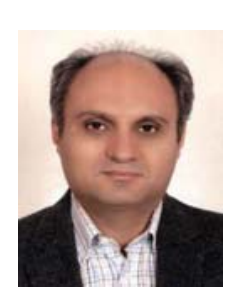

Kamran Farnian was born in April 21, 1970 in Tehran, Iran. He received his B.Sc. degree in mechanical engineering in 1993 from central branch of Islamic Azad University, Tehran, Iran. He has 19 years of experience in industrial management, EPC contracts, and automotive industry. He has held seminars on "After Action Review", "Culture and International Negotiation", and "Institutional Innovation Management” in Iran. He has achieved "Executive Management" and "International Negotiators" certificates. He currently holds the position of CEO at Iran branch of KONE Corporation, Tehran, Iran.

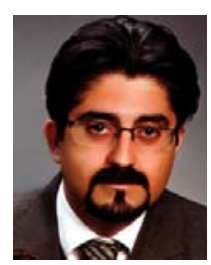

Kourosh Tahouri received his B.Sc. degree in industrial engineering in 1994 from south branch of Islamic Azad University, Tehran, Iran. He has more than 18 years of experience in automotive industry in fields of including balance line engineering, quality control management, and production management. 